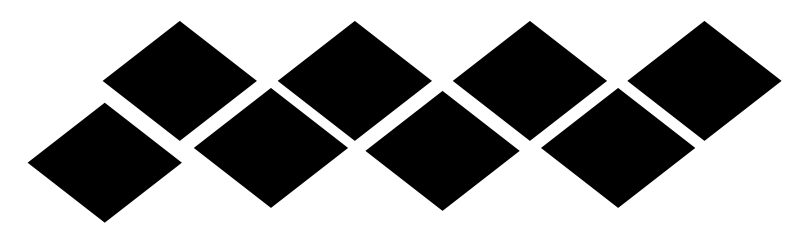

青.

\title{
Le retour impossible et l'incommunicabilité dans la pièce "Juste la fin du monde" de Jean-Luc Lagarce
}

\author{
Préparé par : \\ Alaaedin Baheiedin Alaaedin \\ Professeur adjoint à la faculté des lettres à Quena \\ Université du Sud de la vallée \\ DOI: 10.21608/qarts.2021.89183.1182
}

مجلة كلية الآداب بقنا - جامعة جنوب الوادي - العدد 53 (الجزء الأول) يوليو 2021

ISSN: 1110-614X الترقيم الدولي الموحد للنسخة المطبوعة

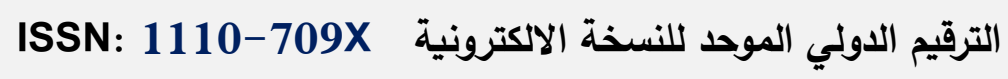
https://qarts.journals.ekb.eg موقع المجلة الاكتروني: 


\title{
Le retour impossible et l'incommunicabilité dans la pièce "Juste la fin du monde" de Jean-Luc Lagarce
}

\author{
Préparé par :
}

Alaaedin Baheiedin Alaaedin

Professeur adjoint à la faculté des lettres à Quena

Université du Sud de la vallée

alaabahi364@gmail.com

\section{Résumé}

Jean-Luc Lagarce est né en (1957), il est aujourd'hui l'un des auteurs contemporains les plus joués en France. Ses textes sont traduits en vingt-cinq langues et sont joués dans de nombreux pays. Dans notre étude, nous avons mis la lumière sur le thème majeur dans la pièce "Juste la fin $d u$ monde": le retour impossible de Louis, le héros principal dans la pièce et son impuissance à déclarer la nouvelle de sa mort jusqu'à la fin de la pièce. Egalement, nous avons mis en évidence le sujet de l'absence de communication entre les personnages de la famille. Nous avons traité également des thèmes subordonnés au thème essentiel de notre étude comme: l'oubli, l'attente, la répétition, et surtout le thème du passé dominant ainsi que les relations familiales. Enfin, nous nous sommes intéressés à montrer les particularités de la langue dramatique de Lagarce qui se distingue par sa désinvolture entre le lyrisme et la langue triviale.

Les mots clés: Le retour impossible, l'incommunicabilité, la pièce "Juste la fin du monde", de Jean-Luc Lagarce 


\section{Introduction:}

Jean-Luc Lagarce est né à Héricourt (1957). En (1975), il s'inscrit au conservatoire d'art dramatique et à la Faculté de Philosophie de Besançon. Auteur et metteur en scène, il met en scène des écrivains comme Ionesco, Marivaux, Labiche, puis ses propres textes. Après une expérience dans le domaine théâtral et son parcours de l'Est de la France et la capitale, il s'installe à Paris à la fin des années (1980). Ses mises en scène des pièces classiques ont réalisé un grand succès plus que ses propres pièces.

Lagarce a écrit vingt-cinq pièces de théâtre, trois récits, dix-neuf cahiers de journal intime, son œuvre a été traduite en vingt-cinq langues et s'impose comme politique et existentielle. En écrivant son œuvre, il vise à dire la vérité, à avoir la capacité de disparaître et enfin à apprendre à mourir.

En (1990), il reçoit le prix Léonard-de-Vinci et part trois mois à Berlin où il rédige "Juste la fin du monde". A son retour en France, très peu sont ceux qui comprennent et acceptent cette pièce qui est entrée au répertoire de la Comédie-Française en (2008) et s'inscrite au programme de l'agrégation de lettres modernes, de lettres classiques et de grammaire, pour la saison de 2012.

Lagarce a achevé "Le Pays lointain" une semaine avant sa mort, dans une tentative de réécriture en livrant une version plus ample et complète de "Juste la fin du monde" après son dernier échec.

La langue dramatique de Lagarce dans ses pièces se caractérise par sa désinvolture entre le lyrisme et la langue triviale. Ses personnages reprennent sans cesse leurs répliques en les modifiant, c'est pourquoi nous remarquons que le texte lagarcien devient de plus en plus hésitant entre le lyrisme et la langue quotidienne.

Une grande partie de son écriture théâtrale est autobiographique. La plupart de ses pièces et surtout sa pièce "Juste la fin du monde" qui est la base de notre étude, traite essentiellement le thème du retour et des adieux avec ses éléments subordonnés : le passé dominant, les relations familiales, l'impuissance à se communiquer avec les autres, et surtout sa langue spéciale utilisée dans la pièce... éléments liés au thème du retour qui sera la problématique essentielle de notre étude. 
Lagarce meurt à trente-huit ans du SIDA. Il s'est condamné sept ans avant sa mort, et concentre son écriture sur la disparition et la famille. Après sa mort, François Barreur œuvre à la reconnaissance de ses écrits, qui ne se produisent qu'à la fin des années 1990.

\section{I-Particularité de l'écriture dramatique de Lagarce}

Si Jean-Luc Lagarce n'a pas été reconnu de son vivant comme un écrivain important, c'est parce que: "le langage théâtral était trop en décalage, trop novateur" (Thibauat, 2008: 7). Lagarce est considéré aujourd'hui l'un des dramaturges français les plus connus et les plus joués non seulement sur les scènes françaises, parmi lesquelles, la Comédie-Française, mais également dans le monde entier, son théâtre est un:

"théâtre de paroles, d'un théâtre presque clos à force de se désintéresser du monde, d'un théâtre hanté par la seule menace de la mort, d'un théâtre de la perte, avant tout." (Brun, 2009: 183).

$\mathrm{Si}$ nous voulons comprendre la dramaturgie de Jean-Luc Lagarce, il faut savoir tout d'abord que "La dramaturgie, en réalité, se dissout dans la textualité: on distingue mal à présent l'action, les conflits, l'espace et le temps, comme si ces catégories perdaient, dans ce type d'écriture, toute consistance ou... se reformaient en d'autres termes." (Pavis, 2011: 184).

Son théâtre se caractérise par examiner les difficultés et les obstacles des relations avec l'autre, concrétiser les désirs qui nous agitent, affronter l'ambiguïté de la solitude et de la passion humaine, il crée une écriture dramatique très efficace et originale, ce n'est pas uniquement un théâtre de paroles, mais: "de paroles obliques, détournées, évidées, de telle sorte que l'échange..." (Brun, 2009: 192).

Nous pouvons considérer le théâtre de Lagarce comme un théâtre de la distanciation, dans lequel, Lagarce expose la destruction et la reconstruction de ses personnages, car il pousse les spectateurs à méditer et à réfléchir plus qu'à ressentir et éprouver en nous frustrant de l'illusion car son: "œuvre s'élabore et s'aiguise de franchir les limites génériques non seulement d'un texte à l'autre, mais aussi à l'intérieur d'un même texte." (Boblet, 2008: 421). 
Les écrits autobiographiques de Lagarce nous décrivent un jeune auteur qui se caractérise par une grande sensibilité et qui se traduit par un regard distingué sur les hommes. Cet itinéraire autobiographique se développe avec une écriture dramatique.

D'autre part, nous pouvons considérer que la qualité indiscutable de la langue de Lagarce est la dimension fondamentale de cette écriture autobiographique qui est:

"musical, on ne le dira jamais, mais elle témoigne surtout d'une maîtresse peu commune, d'une espèce d'exactitude." (Saint-Pierre, 2001: 108)

Loin de la forme conventionnelle du drame, le théâtre de Lagarce apparaît très loin de la forme épique. Lagarce s'est montré comme une figure d'exception et son grand succès dans le monde entier en témoignage. Sa langue distinguée pouvait peindre et traduire le monde autour de lui avec une universalité magique, c'est pourquoi, nous considérons sa dramaturgie comme un point de vue de son époque. Ses pièces représentent une réflexion de la mentalité de cette période dans laquelle il vit: "dans ce théâtre de l'après-coup qu'est le théâtre de Lagarce, théâtre où le futur semble redoubler le passé, théâtre de reprises et de variations à partir du déjà dit, déjà lu, déjà écrit, théâtre travaillé par la question de la filiation et de l'héritage, seule la parole, avec ses failles, ses manques, ses détours, ses ratés, demeure imprévisible et neuve." (Brun, 2009: 195)

Lagarce a crée ses personnages dans la réalité de leur vie, ils sont toujours en quête permanente d'amour dans une société qui vit un état de destruction où l'amour devient de plus en plus difficile à arriver. En arrivant à la fin, on se heurte au silence qui domine ces personnages qui sont face à face, où ils s'attendent, se désirent et se regardent disparaître en saluant l'un l'autre:

"Sur scène, ces personnages ont de motivations différentes, convergentes ou divergentes." (Jongy, 2009: 161)

Les sujets et les thèmes traités dans l'œuvre dramatique de Lagarce sont représentatifs des courants littéraires et dramatiques, ces 
thèmes traitent également l'ambiance familiale, les événements ordinaires et de la banalité, car:

\begin{abstract}
"ces pièces mettent les liens familiaux, amicaux et amoureux au cour du débat, et les abordent sur le mode du règlement de compte infini, les saisissent au sein d'un processus d'achèvement et de liquidation." (Douze, 2011: 8)
\end{abstract}

D'autre part, la plupart des pièces de Lagarce sont concrétisées sur la formule du dramaturge lui-même qui met en scène: "le récit de l'échec, le récit de ce qu'on voulut être et qu'on ne fut pas, le récit de ce qu'on vit nous échapper..." (Lagarce, 2007: 530)

Il est à remarquer que Lagarce propose un renouveau de l'écriture littéraire en déplaçant la langue et la syntaxe au cœur de sa mise en scène. Il ouvre la voie à un autre théâtre, inspiré par le roman, tout en conservant la tradition dramaturgique fondée sur le dialogue. Son théâtre possède également une langue unique, qui déconstruit le langage et se classe dans le genre contemporain.

En bref, Lagarce nous a offert dans son théâtre des digressions et des corrections qui représentent le langage de tout être humain, il propose sa propre langue, parfaitement humaine.

\title{
II- Caractéristiques de la langue dramaturgique lagarcienne
}

"En parlant, chacun de nous cherche ses mots, et en énumère souvent plusieurs avant de trouver le bon." (Blanche-Benveniste, 2010: 26)

Chez Lagarce, le verbe représente une arme destructive, ce verbe se trouve à l'origine de chaque conflit interpersonnel, il a un pouvoir super, par lequel, nous pouvons entrer en contact avec l'autre parce que:

"la langue parlée, c'est aussi la langue dans laquelle se parlent effectivement les gens; elle est donc évidemment un lien hautement sociologique, représentatif par exemple dans bon nombre des pièces de Lagarce, des relations qu'entretiennent les 
membres d'une même famille." (July et Himy, 2012:

100)

La parole chez Lagarce suffit à explorer les hostilités entre les personnages. Quand nous parlons, nous imposons un jugement, nous mettons l'autre dans une qualification précise, nous le figeons dans un certain état. D'ici, apparaît la volonté des personnages à refuser le dire de leurs interlocuteurs:

"la langue de Lagarce mime les errants, les bégaiements, les fourvoiements de l'oral que le passage à l'écrit tend dans son usage normatif à effacer pour ne retenir que les formulations "définitives", proches, elles, du cliché et de la langue conventionnelle." (Brun, 2009/1: 194)

Lagarce cherche toujours dans sa pièce du réalisme, car: "tous les écrivains pensent être réalistes" (Robbe-Griller, 2006: 135). Il a cherché une langue naturelle et réaliste qui serait le reflet des dialogues, ce langage se convient avec le réalisme cherché par Lagarce dans son écriture, car: "le réel n'est représentable, et c'est parce que les hommes veulent sans cesse le représenter par des mots qu'il y a une histoire de la littérature." (Barthes, 1978: 20).

La langue dramatique lagarcienne se distingue par la variété entre les scènes comiques et les scènes tragiques, de la répétition et également par la variété des tons. La domination du passé est la référence des réalités banales comme: le travail, la maison, les enfants qui lui affirment le rapprochement avec le langage parlé.

D'autre part, ces réalités l'éloignent par le retour sur les mots. L'écriture se donne comme telle et impose son propre rythme et participe en même temps à la construction des personnages. Cette langue indique l'hésitation en essayant de trouver les mots justes, ainsi que la difficulté de trouver d'une parole juste dans le sentiment du manque commun à chaque personnage.

$\mathrm{Au}$ niveau grammatical, les temps verbaux dans l'écriture dramatique de Lagarce aident à dynamiser l'attente, l'absence et le doute. Dans ses pièces qui sont consacrées au retour et à la transformation du passé, c'est le temps présent que l'auteur attribue les 
fonctions du passé, le présent prend les caractéristiques de l'Imparfait, le futur simple devient futur en parlant dans le passé.

Ainsi que les temps qui expriment le passé sont tout près du sens du présent en exprimant des actions dont le déroulement n'est pas achevé, cela se manifeste quand l'auteur exprime de l'attente qui se trouve dans l'ensemble de son écriture dramatique.

Le présent sert à convaincre le lecteur qu'il s'agit d'une action réelle et de courte durée, tandis que le Passé composé est utilisé par l'auteur pour exprimer la vérité. Pour le Futur, Lagarce l'a utilisé pour désigner le prolongement des souffrances.

D'autre part, la répétition est un élément très nécessaire dans le style lagarcien. Elle représente une manière de vie des personnages qui essayent de trouver leur harmonie et de créer des relations avec l'autre, celui-ci est plongé à son tour dans ses propres contradictions, cette répétition peut être considérée comme: "un décalage entre ce qu'on imagine être, et ce qu'on voudrait être, et ce qu'on est." (Godard, 2002: 8).

Donc, les répétitions dans l'œuvre lagarcienne forment une partie importante non seulement du style dramatique de Lagarce, mais également dans la fiction lagarcienne dont le déroulement de l'action devient impossible sans les reprises qui dynamisent l'existence de ses personnages dont la vie n'est qu'un retour infini sur le passé et sur le conflit permanent entre la réalité et le rêve impossible de ses personnages.

Il est à remarquer que l'amplification créée par les répétitions donne à la langue la capacité de faire circuler le sens, tandis que les dialogues forment un chemin unique construisant la seule voix de la pièce dès le début jusqu'à la fin. C'est la voix de l'auteur lui-même qui reflète les tonalités des dramaturges du passé. Les répétitions fréquentes et les multiples reprises des textes lagarciens s'accordent avec la version qui parle des insistances rythmiques qui constituent le discours. Ces multiples répétitions qui s'éparpillent dans le discours lagarcien mettent en doute la parole en la vidant de sens:

"Le secret est au cour même de l'écriture, dans la façon dont les personnages prennent la parole et 
partagent la même quête de l'infinie précision puisqu'ils parlent tous la même langue, celle de l'auteur. La cohérence naît du rythme commun, des jeux du partage et de la reprise, et d'une recherche infinie de l'exactitude." (Ryngaert, 2007: 132).

Donc, la parole qui est au centre de la dramaturgie représente le moteur de l'action qui établit l'œuvre lagarcienne: "pour Lagarce, le travail sur la langue est primordial..." (Kwashin, 2006: 79). Dans le théâtre de Lagarce, le drame consiste essentiellement à la parole avec tous ses niveaux: la parole excessive, la parole déplacée et la parole que nous attendons mais qui n'arrive jamais.

En ce qui concerne le langage utilisé par Lagarce dans sa pièce "Juste la fin $d u$ monde", il a utilisé un langage dramatique pour raconter l'histoire de Louis. Pour montrer le trouble de la tension dans la pièce, Lagarce a utilisé des verbes dans diverses formes, tantôt au Présent ou Futur; tantôt au Conditionnel.

Nous remarquons également que les personnages répètent la même phrase plusieurs fois. Ce genre d'écriture nous rappelle l'écriture de Beckett qui utilise parfois des répétitions des phrases courtes dans son œuvre. Celles-ci créent à leur tour une activité dans le déroulement de la pièce en évitant l'effet ennuyeux des soliloques et des monologues.

\section{III- Le retour impossible dans "Juste la fin du monde"}

Dès les années 1980, le thème du retour caractérise le théâtre de Jean-Luc Lagarce. Dans la pièce "Juste la fin du monde", Lagarce met en scène le retour de Louis dans son village, chez sa famille, il est arrivé pour déclarer sa mort prochaine, mais au terme de discussions nerveuses et querelleuses, Louis repartira sans avoir rien dit:

"Le drame théâtral sans projection vers l'avenir accrô̂t le poids du passé et attire le récit." (Boblet, 2008/4: 82).

La pièce se caractérise par son ambiguïté en nous présentant un sujet déjà connu dans le domaine littéraire, c'est le retour du fils qui a quitté sa famille il y a longtemps et revient sur ses pas, plein de 
désespoir et mélancolie pour mourir simplement. Il a voulu annoncer à sa famille sa mort, cette pièce est:

"introduite par le locuteur, déjà mort. La voix que

l'on entend est d'outre-tombe." (Jongy, 2009 : 164).

Dans "Juste la fin du monde", nous apercevons la mixture des genres dramatiques qui s'accorde avec le dérèglement des fonctions psychiques et s'accorde également avec les troubles causés par la déclaration de sa propre mort. La poétique de ces genres s'ajuste avec une sémantique de la forme: "axiologiquement rapporté à un contenu." (Bakhtine, 1978: 82).

La pièce est une œuvre autobiographique qui: "développe; comme une épreuve, les traces mnésiques du vécu" (Ortel, 2002: 310). L'autobiographie n'est qu'un itinéraire ou trajet de révélation, cela se manifeste par excellence dans le cas de Lagarce qu'on peut le considérer l'auteur de pièces autobiographiques.

Lagarce a suit dans "Juste la fin du monde" les intuitions de Roland Barthes, c'est pourquoi, il nous a créé une très belle image de soi sans obscénité ni opacité, en croyant que le "je" est le pronom convenable de l'imaginaire et de la construction esthétique:

"L'imaginaire vient à pas de loup, patinant en douceur sur un passé simple... un souvenir, tout ce qui peut se rassembler sous la devise même du Miroir et de son image: "moi, je"." (Barthes, 1995: 109)

Après un retour du voyage à La Haye, Lagarce commence à se demander: comment vivre avec soi-même et avec les autres en assurant qu'il va mourir bientôt? Il a exprimé tous ses émotions et ses sentiments dans un article intitulé "Le Voyage à La Haye" en disant:

"Raconter le Monde, ma part misérable et infime du Monde, la part qui me revient, l'écrire et la mettre en scène, et construire à peine une fois encore, l'éclair, la dureté, en dire avec lucidité l'évidence. Montrer sur le théâtre la façon exacte qui nous saisit parfois les hommes et les femmes tels qu'ils sont, la beauté et l'honneur de leurs échanges et la mélancolie aussitôt qui les prend lorsque cette beauté et cette horreur se 
perdent, s'enfuient et cherchent à se détruire ellesmêmes..." (Lagarce, 1994:79)

Le message dans "Juste la fin du monde" a totalement disparu, les textes de Lagarce causent toujours un malentendu, car ces textes: "racontent des histoires, et ces histoires fournissent des pistes de sens à l'interprétation." (Ryngaert, 2007: 134). Nous trouvons peu d'actions, mais beaucoup de discours, il n'y a pas beaucoup d'événements qui se produisent, mais l'essentiel dans la pièce consiste sur les échanges verbaux des personnages dans une maison familiale. Dans le début de la pièce, Louis déclare son prologue qu'il va mourir et cette mort est irrémédiable.

Malgré l'absence de message dans la pièce, elle suscite toutes ces questions avec une sorte d'élégance qui agite les points de vue, en bref, le texte consiste essentiellement sur la qualité des échanges verbaux des interlocuteurs.

La structure de la pièce "Juste la fin $d u$ monde" est très intéressante. Elle peut appartenir à celle d'un spectacle de music-hall. Dans la pièce, il y a un narrateur qui raconte et présente les différentes péripéties de l'œuvre et se raconte également, puis il essaie de diriger les autres événements.

\section{1-Les conceptions différentes du titre}

"Juste la fin du monde pourrait donc bien être "Juste la fin du théâtre de l'absurde", juste le début d'un nouveau théâtre, juste le début d'une nouvelle représentation du réel, "juste le début d'un monde" un peu moins tragique." (Gallon, 2012: 77)

Le titre "Juste la fin du monde" fait entendre l'écho de l'expression orale "c'est pas la fin $d u$ monde". Dans ce titre énigmatique, pas d'indication de personnage ni d'action, c'est une attente très ouverte qui explore l'idée de fin; on se demande si c'est une fin ou non? C'est une fin universelle d'un monde en général ou une fin d'un monde en particulier?

Le premier mot dans le titre de la pièce "juste" et dont plusieurs sens rendent compte de plusieurs aspects essentiels de l'œuvre lagarciene, cela consiste sur l'analyse de principales occurrences du 
mot dans la pièce, et à commencer par le titre lui-même, qui pose aussitôt problème. Le terme "juste" au début de la pièce, est tout à fait fréquent chez Lagarce, on entend sûrement "juste" qui fait comprendre le désir et la possibilité de trouver pour chaque personnage la parole juste ou exacte.

D'autre part, le titre "Juste la fin du monde" fait entendre la lourdeur de l'événement à venir. Le titre porte également un aspect pratique qui laisse ressortir l'imminence d'une catastrophe.

En lisant profondément le titre de la pièce, nous remarquons que les lecteurs s'arrêtent pour comprendre le contenu de la pièce, alors, le litre n'est pas venu au hasard, mais l'auteur l'a inventé exprès pour créer un lien solide avec le contexte.

"Juste la fin du monde" est également un titre dérisoire, car il caractérise la personne principale de la pièce, Louis avec sa vie qui s'approche de sa fin inéluctable, mais ce n'est pas la fin de toute l'humanité, c'est un titre ambigu, parce qu'il porte plusieurs significations contradictoires.

D'autre part, le titre porte également deux significations possibles. Premièrement, la notion de "Monde" et de "fin du Monde" se distinguent par leurs dimensions dramatiques qui nous conduisons à l'épique, tandis que la situation qui évoque "la fin du monde" nous mène à un état tragique et hostile:

"Le titre dans son ensemble peut donc en fait être lu tantôt comme un euphémisme tentant de relativiser la situation... tantôt comme une litote ironique et désespérée montrant toute l'atrocité de la situation." (Gallon, 2012: 45)

Par son titre, la pièce "Juste la fin $d u$ monde", évoque la fin inéluctable. Ce titre qui porte une dimension spatio-temporelle, "fin $d u$ monde" qui signifie "bout du monde", et la dimension temporelle qui évoque la fin proche de la vie de Louis. Le geste narratif représenté par Louis lui-même cache le silence intérieur à la représentation et qui fait entrer le monologue final dans une dimension plus dramatique.

\section{2-Une étude intertextuelle de la pièce}


Pour comprendre la pièce "Juste la fin du monde", nous pouvons la mettre dans un cadre intertextuel car: "tout texte se construit comme mosaïque de citations, tout texte est absorption et transformation d'un autre texte. A la place de la notion d'intersubjectivité s'installe celle de l'intertextualité..." (Kristeva, 1969: 84). D'autre part, Gérard Genette affirme cet avis par une autre définition de l'intertextualité qu'elle considère comme une catégorie contenant l'allusion:

"Je définis (l'intertextualité), pour ma part, de manière sans doute restrictive, par une relation de coprésence entre deux ou plusieurs textes, c'est-àdire... par la présence effective d'un texte dans un autre." (Genette, 1982: 8)

Avec "Juste la fin du monde", Lagarce n'hésite pas à faire signe à plusieurs intertextes mythiques qu'il reprend. La pièce se distingue des autres pièces de Lagarce par sa référence et par sa relation intertextuelles.

Au début, nous remarquons la référence religieuse de la pièce, qu'on découvre une relation très forte avec l'histoire de "l'Enfant prodigue" qui est l'une des paraboles donnée par Jésus de Nazareth, également appelée parabole du "Fils perdu". Le récit nous raconte l'histoire des trois personnages: le Père, le Fils aîné, qui obéit sincèrement les ordres et les instructions de son père et ne le quitte jamais, et le deuxième fils, le Fils cadet ou l'enfant prodigue qui, s'éloigne de son père et part pour découvrir le monde et ses merveilles. Ensuite, il a décidé de retourner vers son bon Père qui l'aime toujours. Le Père était toujours joyeux par le retour de son fils cadet. Mais le fils aîné n'était pas satisfait de ce retour. "Juste la fin du monde" a des relations fortes, non seulement avec l'histoire du "Fils prodigue", mais également avec "En attendant Godot" de Samuel Beckette.

L'histoire du fils prodigue existe depuis longtemps dans la littérature et la pensées humaines, la pièce "Juste la fin du monde" n'est qu'une étape de ce drame déjà connu depuis les temps les plus reculés. Contrairement au texte religieux, ici dans la pièce lagarcienne, la Mère replace l'existence paternelle. Vincent Tasselli affirme que:

"le dramaturge s'inscrit dans un contexte chrétien.

Les personnages possèdent, en effet, des noms 
bibliques: Suzanne, Catherine correspondent à des noms de Saints... Louis, lui, est isolé, car il pourrait s'appartenir à Saint Louis, roi de France sans auréole." (Tasselli, 2014: 10)

Cette pensée est déjà reprise dans la pièce à travers le discours qui s'est déroulé entre Catherine et Antoine:

"Catherine: Il porte avant tout le prénom de votre père et fatalement, par déduction...

Antoine: Les rois de France" " (Juste la fin du monde: 18)

La tension entre les deux frères dans les deux histoires est pareille. Dans la pièce de Lagarce, Antoine, le frère de Louis déclare à sa famille que Louis ne mérite d'être bien accueilli en posant cette question qui le hante: Pourquoi il y revient? Il commence à attaquer son frère poussé par la jalousie, malgré de longues années de l'absence de son frère. Nous remarquons les mêmes troubles qui dominent le frère aîné dans l'histoire du "Fils prodigue", après le retour de son frère le plus jeune qui a quitté également la maison et commence à vivre une vie loin de sa famille en dépensant tout ce qu'il avait de l'argent.

Alors, nous avons deux fils dans les deux histoires qui ont quitté leur maison et retourné encore une fois après plusieurs années à leurs maisons, nous ne connaissons pas à travers ces histoires ni les causes de leur départ ni les causes de leur retour. Seulement, Louis dans "Juste la fin $d u$ monde" est revenu pour déclarer qu'il va mourir prochainement, tandis que le fils cadet de "Fils prodigue" est revenu à son père en lui déclarant qu'il a commis un péché contre le ciel et contre lui, et avoue aussi qu'il ne mérite pas d'être son fils.

Donc, la raison principale de ce conflit entre les frères dans les deux histoires, c'est la jalousie. Dans la pièce de Lagarce, la Mère cherche un essor pour arrêter la jalousie d'Antoine en demandant l'aide et l'appui de sa femme Catherine. Mais les rapports entre les frères et la Mère sont devenus trop complexes. C'est une brève comparaison entre les deux histoires à travers le cadre intertextuel qui représente:

"la perception par le lecteur de rapports entre une cuvre et d'autres... Ces autres ceuvres constituent l'intertexte de la première." (Riffaterre, 1980: 4) 
Un autre exemple, à travers lequel, nous pouvons interpréter les dimensions mythiques de la pièce lagarcienne en faisant une comparaison avec le chef-d'œuvre d'André Gide "Le retour de l'enfant prodigue". En lisant la pièce de Gide, nous assistons à la même tension entre les frères, mais la seule différence, c'est que Gide a ajouté un autre frère. Dans la pièce de Gide, le frère aîné réfléchit exactement comme Antoine dans la pièce de Lagarce, il voit que son frère qui est revenu après de longue date, ne mérite d'être accueilli fidèlement, parce que ce frère a choisi son destin librement, et c'est sa propre faute. Tandis qu'Antoine dans "Juste la fin du monde" a essayé de convaincre sa famille que Louis a commis une grande faute en quittant la maison familiale.

Mais ce qui distingue l'œuvre de Lagarce des autres histoires que nous venons de proposer, c'est l'originalité de Lagarce qui réside dans la création d'un monde tout à fait différent en utilisant dans sa pièce les monologues, les quasi-monologues et les soliloques en même temps.

Dans le cas des quasi-monologues, nous trouvons une personne qui parle en racontant une histoire ou exprimer une certaine idée, on l'écoute sans réponse, le soliloque ressemble le quasi-monologue, ils portent tous les deux la même conception et qui vise à orienter la parole à un interlocuteur présent sur la scène, mais qui reste toujours en état de silence voire mutisme. Selon la définition de Bakhtine, le soliloque est: "une attitude dialogique à l'égard de soi-même. C'est une conversation où l'on est son propre interlocuteur." (Bakhtine, 1970: 16).

\section{3-Le passé dominant}

Ce qui caractérise la dramaturgie lagarcienne consiste dans le fait que nulle action ne se déroule dans le présent de la représentation dramatique. Dans la pièce, Louis est le messager de sa propre mort. Il concrétise la seule action de la pièce: c'est l'action du dire et du nondire, tandis que le thème essentiel dans la pièce, c'est le retour difficile de Louis dans l'univers lointain du passé et la coexistence impossible avec une famille devenue lointaine.

La pièce oscille entre le geste narratif de Louis et le dialogue conflictuel avec les autres membres de la famille qui est devenu un dialogue dramatique. Mais Louis n'est plus un personnage dramatique, 
car il se transforme à un personnage collectif, il n'est qu'un être de parole qui adressé sa parole dans une langue commune à tous les personnages.

Les pièces de Jean-Luc Lagarce contiennent des personnages en train de revisiter leur passé, c'est le passé qui domine l'imagination de toute l'œuvre dramatique de Lagarce. Il représente le facteur essentiel et dynamisant du déroulement de chaque histoire, car le temps du récit ne représente qu'un lien entre un passé qu'on ne peut se débarrasser et un présent dans lequel les personnages essayent de remettre en action et d'actualiser ce qui s'est déjà déroulé dans le passé.

Ce retour dans le passé est lié étroitement au sujet de l'oubli qui permet aux personnages de nier complètement ce passé difficile et fatigant. L'oubli est un moyen volontaire ou involontaire des cerveaux des personnages qui leur aide à se débarrasser des moments tristes et difficiles ou de les changer en fournissant leur propre point de vue des choses. L'oubli est une manière très confortable pour les personnages de cacher ou de défigurer les vraies intentions du langage.

Dans "Juste la fin $d u$ monde", l'oubli n'est qu'une manière adoptée par les membres de la famille de Louis pour exprimer leur indifférence concernant le sujet de départ de Louis qui ne leur a apporté beaucoup de souffrances; cet oubli est exprimé par Suzanne; sœur de Louis en disant:

\section{"Lorsque tu es parti}

Je ne me souviens de toi-

Je ne savais pas que tu partais pour tant de temps, je

n'ai pas fait attention, je ne pensais pas garde,

et je me suis retrouvé sans rien.

Je t'oubliai assez vite.

J'étais petite, jeune, ce qu'on dit, j'étais petite." (Juste la fin du monde : 21)

Dans cette phrase ci-dessus, Suzanne nous raconte le fait qu'elle ait oublié son frère à son âge. Elle affirme également qu'elle avait beaucoup perdu en oubliant son frère car il occupait pendant son absence, la place d'une idole admirée par elle-même et pour tous les membres de la famille. 
Quant à Louis, ce retour a éveillé les souvenirs d'une autre vie qu'il a quittée depuis longtemps. Son impuissance à achever sa mission pour laquelle il est revenu à sa famille est contradictoire avec sa volonté de se souvenir et de plonger dans un univers qu'il a préparé dans les profondeurs de son mémoire.

Il est à remarquer que la pièce se termine sur un monologue dans lequel, Louis raconte en détails une des péripéties de sa vie, où il n'a pas fait ce qu'il rêvait et qui aurait pu amener des moments de joie. Il a fait une comparaison entre cet épisode raconté par lui et son retour manqué. Louis regrette d'oublier un événement de sa vie, il admet son oubli:

"Louis- Après, ce que je fais, je pars.

Je ne reviens plus jamais. Je meurs quelques mois plus tard, une année tout au plus.

Une chose dont je me souviens et que je raconte encore (après j'en aurai fini).

C'est l'été, c'est pendant ces années où je suis absent...

Je me remets en route avec seul le bruit de mes pas sur le gravier. Ce sont des oublis comme celui-là que je regretterai" (Juste la fin du monde :94)

Donc, l'oubli de Louis représente un refus de la réalité objective avec toutes ses souffrances et ses maux, c'est un révolté intérieur visant à construire une réalité subjective. Dans cette réalité tellement cherchée, le personnage peut être celui qu'il rêve. Dans l'écriture lagarcienne, l'oubli est une manière efficace de changer le passé en créant un lien entre l'acte de parole et l'acte d'oubli.

Les personnages tragiques dans la pièce souffrent de l'oubli, l'oubli de leur passé, et qui va jusqu'à oublier une personne très proche d'eux. Ils ont des trous de mémoire qui seront remplis par les autres. Donc, ce refus de la réalité n'est qu'un désir intérieur qui nie la vie avec ses malheurs, où chaque personnage cherche l'être qu'il veut.

Le refus de la réalité chez les personnages est concrétisé dans la pièce "Juste la fin $d u$ monde" dans le phénomène de l'attente qui accompagne toujours la parole prononcée. Avec cette attente, les personnages refusent cette réalité objective en la substituant par la 
réalité subjective qui devient de plus en plus vivante grâce à l'activité de parole.

L'effet de l'attente qui domine la pièce dès le début jusqu'à la fin est claire. Dans la première partie de la pièce, c'est-à-dire dans le prologue, on connait tous que Louis va mourir prochainement, c'est pour cela, il a décidé de revenir chez lui, juste pour annoncer sa mort, tandis que, nous, les lecteurs/spectateurs attendent ardemment le moment convenable où Louis va expliquer à sa famille cette nouvelle en se demandant: qu'est-ce qu'ils font, les membres de la famille, en écoutant cette nouvelle? Au cours de la pièce, l'attente s'accroît de plus en plus pour les lecteurs, ainsi que pour Louis lui-même.

D'autre part, pour Louis, l'angoisse est liée à une forme de retour vers l'enfance: "Je suis enfant" (Juste la fin du monde:67) en trouvant les souvenirs de l'enfance et surtout la chanson: "Je me le chantonne pour entendre juste le son de ma voix" (Juste la fin du monde:67), c'est une chanson qui certainement s'oppose à l'amour chantée par la Mère qui porte des couleurs funèbres.

Dans "Juste la fin du monde", nous remarquons un mélange de temps: "Je pense, je pensais" (Juste la fin du monde:88), et "Aujourd'hui ce n'est rien, ce n'était rien" (Juste la fin du monde:89). Antoine est occupé par le souvenir en montrant l'influence du passé sur lui: "mais je garde cela surtout en mémoire" (Juste la fin du monde:90). Les souvenirs du passé concernant Louis restent vrais, mais selon lui se passent ailleurs:

"Nous pensons, et beaucoup de gens, ... beaucoup de gens doivent assurément le penser aussi, nous pensions que tu n'avais pas tort, nous pensions que en effet, nous ne t'aimions pas assez, ou du moins, que nous ne savions pas le dire." (Juste la fin du monde:89)

Alors, nous pourrions présupposer une temporalité au présent chez Lagarce où Sarrazac nous rappelle que:

"l'une des principales caractéristiques du drame moderne et contemporain, c'est le glissement progressif de la forme dramatique de ce statut primaire. L'action ne se déroule plus dans un présent 
absolu, comme une course vers le dénouement, mais consiste de plus en plus en un retour... sur un drame passé et d'un catastrophe toujours déjà advenue." (Sarrazac, 1995: 12)

\section{4-Les rapports familiaux}

En examinant la pièce, nous remarquons qu'elle contient cinq personnages: Louis, le héros, Antoine, son frère, Suzanne, sa sœur, la Mère et Catherine, femme d'Antoine; mais nous ignorons le prénom de la Mère. Cette pièce est divisée en deux parties avec un prologue au début de la pièce et l'épilogue à la fin. La première partie implique onze scènes, tandis que l'intermède se compose de neuf scènes. Quant à la deuxième partie, elle contient seulement trois scènes.

L'action dans la pièce traite les rapports familiaux, dans une distribution restreinte des personnages dans un lieu unique, un huis clos familial tournant autour du personnage de Louis. Nous avons une situation très particulière de la Mère, qui n'a pas de prénom; c'est une vision double à la fois réaliste par les prénoms des personnages, mais en même temps symbolique par le jeu de rapports mis en place.

L'espace scénique qui est la maison de la Mère et de Suzanne exprime une distribution des personnages par rapport au lieu: Antoine/Catherine, la Mère/Suzanne, ce qui singularise le personnage de Louis, tandis que le temps de l'action se situe dans un temps référentiel, celui des réunions familiales: "un dimanche évidemment", mais s'ouvre sur une autre durée: "ou bien encore, près d'une année entière" (Juste la fin du monde:8)

Au début de la pièce, le prologue est plein d'ambiguïtés mais répond aux besoins du début d'une œuvre théâtrale. Le prologue porte des informations sur le personnage de Louis: son âge, sa mort prochaine, son caractère, le rapport à la parole, son exigence de la liberté et son désir de maîtrise. Ces informations concernant l'ordre psychologique sont en partie avec la manipulation de sa parole que se montre aussi la structure du prologue dans l'utilisation grammaticale de la phrase qu'il donne de lui: "paraître" et "donner aux autres une dernière fois l'illusion d'être responsable de moi-même" (Juste la fin du monde: ) 
L'action elle-même exprime un retour: "retourner les voir" (Juste la fin du monde:6), "revenir sur mes pas" (Juste la fin du monde:6 ) et "aller sur mes traces" (Juste la fin du monde:6), ce retour est dans le temps et dans l'espace, c'est pourquoi, l'action se réforme sur elle-même, elle consiste sur l'acte de Dieu. D'autre part, le prologue met en évidence une relation entre le lecteur/spectateur et le personnage de Louis en donnant une dimension tragique de la pièce.

Ainsi, dans son ambiguïté, le prologue nous met dans une situation tragique face à un personnage qui refuse cette situation tragique, sa parole et son annonce allaient lui permettre de surmonter la mort, et finalement, la relevait de sa propre volonté.

Suzanne, la seule sœur de Louis représente le désir de vivre ailleurs: " je voudrais partir" (Juste la fin du monde : 25", mais ce désire ne se réalise pas: "Je ne pars pas :25" (Juste la fin du monde" et sa vie continue "Je reste", "Je vis où j'ai toujours vécu" Juste la fin du monde:25" et "J'ai ma chambre, je l'ai gardée" (Juste la fin du monde:25), cette continuité représente un destin constant qui ne change pas: "Peut-être que ma vie sera toujours ainsi" (Juste la fin du monde:25), elle représente un modèle pour tous les personnages, sa vie est un mode d'appartenance au groupe:

"Il y a des gens et ils sont le plus grand nombre/il y a des gens qui passent toute leur existence là où ils sont nés." (Juste la fin du monde:25)

Le malheur et l'ennui qui enveloppent les propos de Suzanne proviennent de son désir qui se heurte à de nombreux obstacles, contre lesquels, elle n'a pu lutter. L'espace est l'un de ces obstacles puisqu'il est plus grand que celui d'un studio où elle peut loger indépendamment: "beaucoup mieux que ce que je pourrais trouver avec l'argent que je gagne." (Juste la fin du monde:26). Les choses représentent aussi un obstacle pour elle: "J'ai aussi des choses qui m'appartiennent, les choses ménagères" (Juste la fin du monde:26). Mais le désir réel de Suzanne réside dans la puissance d'affranchir l'existence familiale et d'avoir un espace à soi. La difficulté pour Suzanne se trouve dans sa vie tragique qui ne lui appartient vraiment.

Nous remarquons également que Louis commence sa parole dans la pièce par "pas plus tard", suivi toujours par "l'année d'après", 
ce procédé empêche d'approcher l'événement aux autres membres de la famille et surtout quand il annonce: "J'allais mourir à mon tour" (Juste la fin du monde:6 ). Mais le retour annoncé par Louis ne crée que d'autre séparation avec les membres de la famille, un nouveau départ apparaît avec la déclaration de la mort annoncée aux spectateurs. Donc, cette venue de Louis n'est qu'un retour, rien de neuf et rien n'arrive.

La question du retour qui hante toujours Lagarce et surtout dans ses dernières pièces ne contient pas seulement une intervention du passé dans le présent, mais qui demande une répétition généralisée:

"La mort réelle, la mort symbolique (la séparation) ou le terme de la parole n'existent que projetés dans un futur plus ou moins lointain et atteignable." (Brun, 2009: 185).

Dans la pièce "Juste la fin du monde", la question du retour n'est pas liée au sujet de la distance où apparaît la tension entre "l'étranger et le familier" (Sarrazac, 1999: 150) et qui serait une exhortation à reconnaître la vérité, mais qui est selon la parole de François Jullien une:

"force de reconnaître que ce goût injustifié du retour n'en constitue pas moins une sorte de talent, que cette dérobade continuelle... s'est érigée en élégance..." (Julien, 1995: 48).

Les membres de la famille dans la pièce cherchent toujours à être ailleurs. Suzanne veut aller très loin et vivre une autre vie tout à fait différente, Antoine ne veut pas être là, tandis que Louis qui vient d'arriver paraît toujours avoir ce désir de partir:

"tu étais à peine arrivé... et tu aurais voulu aussitôt repartir." (Juste la fin du monde:7).

C'est pourquoi, on se heurte beaucoup aux questions qui concernent le départ ou l'arrivée dans la pièce, car tout le monde désire quitter leur place et chercher une autre: "Où est-ce que tu vas" (Juste la fin du monde:29), "Où est-ce que vous allez" (Juste la fin du monde:71) et "Où est-ce qu'ils sont" (Juste la fin du monde:30), toutes ces questions nous mettent dans un jeu d'aller et retour ou dans un jeu de cache-cache. 
Donc, la seule chose que nous trouvons drôle dans la pièce, c'est la présence des personnages qui se voudraient ailleurs, cette présence apparaît insupportable malgré qu'elle est longtemps espérée, mais cette présence a bougé une volonté de fuite en se souvenant le premier départ. La présence s'accompagne toujours dans la pièce avec l'absence. Le retour de ces personnages n'est pas uniquement dans l'espace, mais également dans le temps, ce sont des: "longs détours pour juste recommencer." (Juste la fin du monde:32).

Lagarce apparaît influencé par les mythes qu'il reprend dans sa pièce où Louis se montre un pitoyable messager qui arrive et repart sans avoir déclaré sa mort prochaine; Lagarce s'intéresse à jouer avec les conflits fraternels qui se déroulent entre les deux frères: Louis et Antoine.

Nous apercevons également dans la pièce les multiples mésententes entre les personnages qui expliquent la présence fréquente des monologues qui se dispersent dans plusieurs scènes, ainsi que les discours clos sur eux-mêmes, et pour se comprendre:

"il ne faut pas de se parler. Ce qui frappe dans Juste la fin du monde, c'est la propension des personnages à dire ce que les autres ne souhaitent pas entendre autant qu'à taire ce qui est attendu d'eux." (Brun, 2009: 190).

Les discoures entre les personnages se trouvent dans un style indirect, la Mère, c'est elle qui transporte la parole de Louis aux autres, et en même temps, elle dit à Louis ce que son frère et sa sœur attendent de lui, Suzanne, lui informe la douleur la douleur de la Mère après son départ, tandis que Catherine refuse de jouer ce rôle d'intermédiaire auprès d'Antoine qui dit:

"Ne me dites rien, je vous interromps, il est bien préférable que vous ne me disiez rien et que vous lui disiez à lui ce que vous avez à lui dire." (Juste la fin du monde:38).

\section{5-Absence de la communication}

La chose la plus importante dans "Juste la fin du monde", c'est l'absence totale de communication, et malgré cette absence, on parle 
toujours à quelqu'un, non pas au chaos, c'est le silence complet qui domine les personnages de la pièce, Anne Ubersfield affirme cet avis dans son article intitulé "La parole solitaire" en citant que la parole:

"s'adresse à quelqu'un, elle est en même temps éloignée de son destinataire par l'absence de communication concrète. La réplique est bien destinée non pas au vide, mais à tel ou tel." (Ubersfield, 2008: 62).

Donc, la parole de la pièce est prononcée dans l'absence totale de communication ou même sans compréhension. Cette parole sert à approfondir l'existence solitaire de chaque personnage qui ne peut continuer sa propre vie ou de faire un échange verbal avec l'autre afin de s'évaluer.

Suzanne, en s'adressant à Louis, elle se montre incapable de déclarer son désir de vivre ailleurs: "Je ne sais comment l'expliquer/comment le dire/alors je ne le dis pas." (Juste la fin du monde:25 ). Elle cherche en louis un allié dans la mesure où il a quitté la maison familiale et occupe un espace inconnu pour elle, également, dans la mesure où il représente une possibilité d'existence qui est contradictoire à la force du groupe. Comme signe d'approche, elle est prête à lui montrer son appartement, c'est-à-dire de l'intervenir dans une part de sa vie privée, mais Louis ne donne aucun signe, il ne répond pas et la laisse parler jusqu'à la fin de sa parole, voire la fin de sa tirade, dans laquelle, elle lui a exprimé son désir. Les derniers mots de Suzanne affirment l'échec de sa demande:

"tu aurais eu tort/en effet/de t'inquiéter" (Juste la fin du monde: 27)

Malgré cela, on ne doute pas que Louis ne comprend pas le désir de Suzanne, la situation dramatique montre qu'il ne peut pas y répondre telle que le prologue l'a énoncé, parce que Louis n'appartient plus à ceux qui ne peuvent choisir leur destin. Dans le prologue, le lecteur/spectateur remarque aussi les signes exprimant de la mort de Louis. La chambre-débarras porte une signification symbolique:

"et la tienne encore si je veux/mais celle-là, nous n'en faisons rien/c'est comme un débarras, ce n'est pas méchanceté, on y met les vieilleries qui ne servent 
plus mais qu'on n'ose pas jeter." (Juste la fin du monde:25-26)

C'est pourquoi, nous remarquons dans la tirade de Suzanne pleine de vitalité et de volonté et qui commence à devenir funèbre qu'elle porte les signes de la mort en s'adressant à un personnage qui attend sa mort.

A cause de l'absence de toute compréhension, l'incommunicabilité a dominé le discours des personnages où chaque personnage est enfermé dans sa solitude. Ces orientations de la scène lui causent la tristesse dans un théâtre de finesse qui consiste sur les mots et le silence.

\section{Conclusion}

Au début de notre étude, nous avons exposé la pièce " $\underline{\text { Juste la fin }}$ du monde" dans le cadre intertextuel; ainsi que ses références historiques et religieuses et surtout l'histoire de l' "Enfant prodigue" citée dans l'Evangile. Ainsi que la ressemblance qu'elle a avec l'œuvre d'André Gide "Retour de l'enfant prodigue".

La problématique essentielle de notre étude était concentrée sur le thème du retour dans la pièce de Lagarce "Juste la fin du monde", c'est le thème majeur qui domine toute la pièce et conduit ses événement dès le début jusqu'à la fin.

Louis, le héros devient un spectateur qui ne peut rien faire qu'attendre la fin du jour ou la fin de l'année plongeant dans un désespoir total. Il se perd dans ces tensions familiales et reste muet en ce qui concerne la question de sa maladie, cela donne à la pièce l'impression qu'on est en présence d'une tragédie contemporaine.

Le thème du retour dans la pièce est lié à d'autres thèmes subordonnés, comme l'oubli, l'attente, la répétition et le passé dominant. Les personnages dans le théâtre de Lagarce ne racontent pas une histoire où il n'y a pas d'intrigue, ils parlent et se souviennent, ils sont là, mais en même temps, ils sont absents, ils sont des êtres ridicules avec passion. Ces personnages sont prisonniers dans le seul acte: acte de parole où ils essaient sans cesse d'oublier leur passé, mais vainement. 
Le passé dans l'œuvre devient le moteur qui pousse ces personnages et devient le seul mode de vie qui existe pour eux. Ils ne s'arrêtent à changer le passé par la parole prononcée en transformant les histoires en des réalités subjectives.

La fiction dramatique offre une image de l'attente constante d'un événement qui arrête la stabilité des vies des personnages. L'attente et la parole découvrent le refus de la réalité objective remplacée par la réalité subjective.

D'autre part, les personnages lagarciens sont affrontés à l'oubli, c'est l'oubli volontaire du passé, dans lequel, les souffrances vont jusqu'à oublier une personne tout près de nous, c'est un révolte qui conduit à une fuite vers la réalité où chaque personnage cherche ce qu'il voudrait être.

La parole dans l'œuvre de Lagarce est l'élément le plus actif, elle s'impose aux personnages et au déroulement de l'action, c'est pourquoi, les personnages lagarciens veulent maîtriser et saisir une parole qui leur échappe et qui rend impossible de faire reconnaissance de l'autre. La compréhension devient très loin, car le passé remonte en établissant une barrière entre les personnages. 


\section{Bibliographie}

\section{I-Corpus}

1-LAGARCE Jean-Luc, "Juste la fin du monde", Besançon, Les Solitaires Intempestifs, 1999.

\section{II-Ouvrages consacrés à Lagarce et son ouvre}

1-LAGARCE Jean-Luc, "Journal 1990-1995", Besançon, Les Solitaires Intempestifs, 2007.

2-RYNGAERT Jean-Pierre, "Juste la fin du monde: dire avec une infinie précision", in Lire un classique du XXe siècle, Besançon, Les Solitaires Intempestifs, 2007.

3-THIBAUDET Jean-Pierre, "Connaissez-vous Jean-Luc Lagarce?", Besançon, Les Solitaires Intempestifs, 2008.

\section{III-Ouvrages généraux}

1-BAKHTINE Mikhaët, "La poétique de Dostö̈vski", Paris, Seuil, 1970.

2---------------, "Esthétique et théorie du roman", Paris, Gallimard, 1978.

3-BARTHES Roland, "Leçon", Paris, Seuil, 1978.

4---one 1995.

5-BLANCHE-BENVENISTE Claire, "Approche de la langue parlée en français", Paris, Orphys, 2010.

6-DOUZE Catherine, "Lectures de Lagarce", Rennes, Presses Universitaires de Rennes, 2011.

7-GENETTE Gérard, "Palimpsestes- La lecture au second degré", Paris, Seuil, 1982.

8-GODARD D., "Traces incertaines", Besançon, Les Solitaires Intempestifs, 2002. 
9-JULIEN François, "Le Détour et l'accès", Paris, Grasset, 1998.

10-KRISTEVA Julia, "Sémétoike", Paris, Seuil, 1969.

11-ORTEL Philippe, "La littérature à l'ère de la photographie", Nîmes, Jacqueline Chambon, 2002.

12-PAVIS P., "Le théâtre contemporain", Paris, Armand Colin, 2011.

13-ROBBE-GRILLER Alain, "Du réalisme à la réalité", Paris, Editions de Minuit, 2006.

14-SARRAZAC Jean-Pierre, "L'Avenir du drame", Circé, 1999.

\section{$\underline{\text { IV-Articles consacrés à Lagarce et son œuvre }}$}

1-BOBLET Marie-Hélène, "L'hybridité générique du théâtre de Lagarce. Le Pays lointain (1995)", in Poétique 2008/4 (No. 156), pp. 421-434.

2-BRUN Catherine, "Jean-Luc Lagarce et la poétique du retour: l'exemple de Juste la fin du monde", Revue d'histoire littéraire de la France, 2009/1 (Vol. 109), pp. 183-196.

3-GALLON Stéphane, "Lagarce. Juste le début de Juste la fin du monde", in Questions de style, No. 9, 2012, pp.37-77.

4-JONGY Béatrice, "Jean-Luc Lagarce, l'écrivain exposé", dans Interférences littéraires, nouvelle série, No. 2, "Iconographies de l'écrivain", mai 2009, pp. 161-174.

5-JULY Joël et HIMY Laure, "Questions de Lagarce", in Questions de style, Caen, Presses Universitaires de Caen, 2012, pp.93-108.

6-KWASHIN Joëlle, "Le Voyage à La Haye, de Jean-Luc Lagarce", in La Revue Nouvelle, No. 11/novembre 2006, pp. 28-79.

7-LAGARCE Jean-Luc, "Le Voyage à La Haye", in Revue d'esthétique, No. 20, mars 1994.

8-SAINT-PIERRE Christian, "Jean-Luc Lagarce, l'éternel amoureux", in Jeu, No. 98; 2001, pp. 104-110. 
9-TASSELLI Vincent, "Marguerite Duras, Jean-Luc Lagarce: le dialogue troué, un geste théâtral contemporain", Loxias, No. 46, Doctoriales XI, 2014.

\section{V-Articles généraux}

1-RIFFATERRE Michaël, "La trace de l'intertexte", in La Pensée, No. 215, Octobre 1980.

2-SARRAZAC Jean-Pierre, "Le partage des voix", in Nouveaux territoires du dialogue", ouvrage dirigé par Jean-Pierre Ryngaert, Actes Sud- Papiers, coll. "Apprendre", 1995.

3-UBERSFIELD Anne, "La parole solitaire", in Jeu: revue de théâtre, No. 10, 2004. 
العودة المستحيلة وانعدام التواصل في مسرحية (إنها مجرد نهاية العالم) للكاتب جون لو لوك لاجارس

$$
\text { إعداد }
$$

علاء الدين بهي الدين علاء الدين

أستاذ مساعد بقسم اللغة الفرنسية وآدابها

$$
\text { كلية الآداب - جامعة جنوب الوادي }
$$

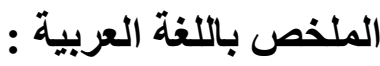

وُلد الكاتب جون لوك لاجارس في عام 190V 19 ويعتبر اليوم أحد الكتاب

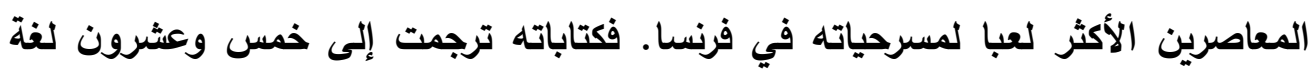

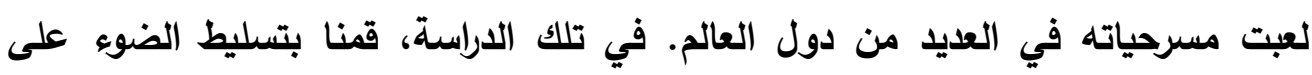

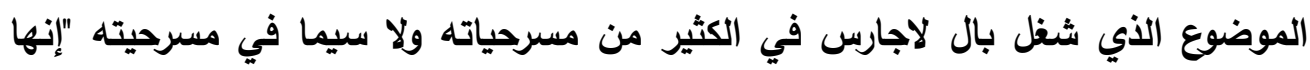

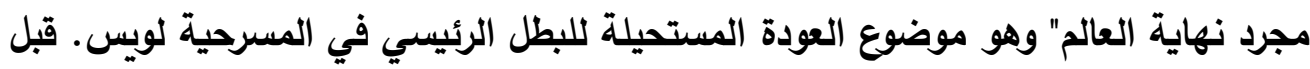
ذلك قمنا بتناول موضوع خصوصيات اللغة الدرامية للكاتب لاجارس والتي تتميز عن غيرها

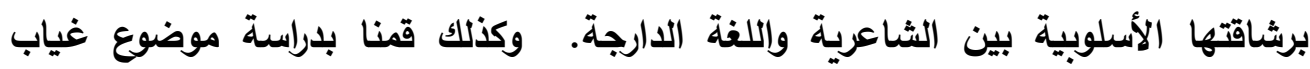

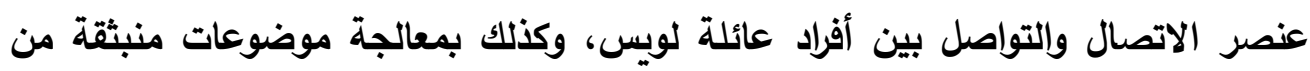

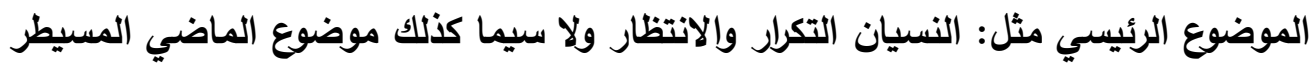
على كل الأثخاص في تلك المسرحية.

، الكلمات المفتاحية: العودة المستحيلة، انعام التواصل، مسرحية "إنها مجرد نهاية العالم"-

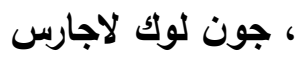

